suggest that a basic concept is developing which relates to an initial stimulus-response coupling in inflammatory cells, subsequently amplified by the formation and release of various mediators. A brief assessment of all the topics included in this book, however, leaves the reader wondering what the hierarchy and relative importance of the various inflammatory mediators might be, and an extended editorial pointing the way to the future would have been most useful. As it is the editors have put together a good book which provides an update on a broad range of recent trends in inflammation, one that makes easy reading for both the neophyte and established researcher.

University Hospital of

DAVID E WOOLLEY

South Manchester,

Manchester M20 8LR

Nonsteroidal Antiinflammatory Drugs. By J G Lombardino. Pp 398. £101·80. Wiley: Chichester, Sussex. 1985.

To paraphrase an old saying of Isaac Asimov, the sound of heavy breathing in the corridors of research institutions should not cause alarm, it is merely the brothers and sisters attempting to keep up with the literature. For those researching in the field, this book Nonsteroidal Antiinflammatory Drugs will bring some relief to the respiratory system.

The volume is more than just a turgid litany of the chemistry and the biology of these compounds. Rather, it consists of four sections dealing with the changes in cartilage in osteoarthritis, the mediators and the mechanisms of inflammation, laboratory methods for testing nonsteroidal anti-inflammatory drugs (NSAIDs), and finally, the medicinal chemistry of NSAIDs.

Together, these four sections form a comprehensive overview and reference to the science of NSAIDs, without entering the field of clinical trials. Perhaps the opening section on articular cartilage, while being quite excellent, appears a little out of context.

The complex subject of mediators and mechanisms of inflammation is well described by Dawson and Willoughby, with the interaction of chemical, cellular, and immunological mechanisms well explained and illustrated.

A large and comprehensive section by Otterness and Bliven describes both the testing of NSAIDs for efficacy, and side effects. The number of models of acute and chronic inflammation described is impressive. The accurate description of particular techniques will be useful for both the beginner in this field and also for old hands who wish to expand their repertoire. Clinicians will find the section useful for assessing the relative merits of data from animal experiments when extrapolation to clinical pharmacology is required.

A section on the medicinal chemistry of the NSAIDs covers the subject in sufficient depth to be useful to chemists and biologists alike. Both familiar and little known NSAIDs are covered in a logical fashion, emphasis being placed on the derivation of each substance from its parent compound, and comparisons are made of the antiinflammatory and analgesic efficacy of comparative structures. This section, like the others, is distinguished by the comprehensive list of references in the text.

The shock of the cost of scientific textbooks never ceases to cause pain; this volume produced the usual reaction. For serious students of the subject, however, the comprehensive and generously referenced text is probably a required purchase; followed swiftly by $600 \mathrm{mg}$ of soluble aspirin (not on an empty stomach).

Senior Lecturer in Pharmacology,

A J COLLINS University of Bath and

Royal National Hospital for Rheumatic Diseases, Bath

\title{
Notes
}

\section{XIth European Rheumatology Congress}

The congress will be held in Athens, Greece from 28 June to 4 July 1987 . Main topics will be adult inflammatory joint diseases; autoimmune connective tissue diseases; juvenile rheumatism; degenerative joint diseases; crystal induced arthropathies; metabolic bone diseases; soft tissue rheumatism and mechanical spine disorders; miscellaneous bone disorders and malignant tumours; orthopaedic intervention; rehabilitation and physical therapy in the rheumatic diseases; and medical-social aspects. Details of the scientific programme from Professor H H Moutsopoulos,
University of Ioannina Medical School, Ioannina, Greece. General information from Horizon, 14 Nikis Street, GR-105 57 Athens, Greece.

\section{Symposium on hand and upper extremity implant surgery}

The 17th annual international symposium on implant surgery for the hand and upper extremity will be held at Blodgett Memorial Medical Center and Amway Grand Plaza Hotel, Grand Rapids, Michigan, USA on 11-13 September 1986. Inquiries to Dr Alfred B Swanson, Blodgett Professional Building, 1900 Wealthy Street, S E, Suite 290, Grand Rapids, Michigan 49506, USA. 